\title{
Better safe than sorry: a potential prophylactic treatment for brain metastasis
}

Many types of cancer metastasize to the brain at the final stage of progression. A recent study by Benbenishty et al. shows that prophylactic administration of CpG-C, a Toll-like receptor 9 (TLR9) agonist, significantly reduces the development of brain metastasis in preclinical mouse models. Such an effect is mediated by the activation of microglial cells that, upon CpG-C treatment, kill and phagocytose the tumour cells during the early stages of invasion into the brain.

Patients with primary tumours such as lung, melanoma and breast cancer have a high potential of developing brain metastasis. Once brain metastases have developed, the current available treatments rarely result in an increased survival. Therefore, a prophylactic approach for patients with cancer at high risk of developing brain metastasis could be extremely advantageous. Intracerebral administration of TLR9 agonists such as $\mathrm{CpG}-\mathrm{C}$ in patients with glioblastoma has yielded promising results in a few phase I/II clinical trials with minimal toxicity. However, such an intracerebral approach would be impractical prophylactically. Hence, the authors set out to test whether systemic administration of $\mathrm{CpG}-\mathrm{C}$ could be a viable strategy as a prophylactic treatment against brain metastasis.

Benbenishty et al. used a model of brain metastasis in which a metastatic cell line is injected into the cerebral circulation of a mouse and then is tested for its ability to seed in the brain and give rise to a tumour. Using both a syngeneic and a xenograft model of non-small-cell lung carcinoma cells, the authors showed that a single systemic prophylactic injection of CpG-C, administered $24 \mathrm{~h}$ before tumour cell injection into the cerebral circulation, was sufficient to reduce brain tumour growth. Such an effect was independent of the adaptive immunity, as the same effect was observed in both the syngeneic and the xenograft models.
The authors confirmed the finding using a more relevant clinical setting. They used a murine model of spontaneous brain metastasis in which melanoma cells are orthotopically injected, resulting in a primary tumour in the flank. Mice were treated systemically with $\mathrm{CpG}-\mathrm{C}$ or vehicle three days before and three days after the surgery to remove the primary tumour. Nine weeks after surgery, mice treated with CpG-C showed significantly reduced cerebral metastatic burden.

Further analysis utilizing isotopelabelled tumour cells in the syngeneic mouse model of cerebral injection showed that $\mathrm{CpG}-\mathrm{C}$ prophylactic treatment affected the number of tumour cells retained in the brain during the early phases of seeding. The effect was similar in males, females and in young, juvenile and old mice and was not altered by chronic stimulation via multiple CpG-C injections.

Natural killer (NK) cells and macrophages were previously shown to mediate the peripheral anti-metastatic activity of TLR9 agonists. However, the authors observed no alterations in the beneficial effects of CpG-C on the brain colonizing ability of tumour cells upon depletion of either NK cells, via anti-NK1 antibody, or macrophages, via clodronate liposomes. However, they found that the CpG-C effect was exerted through a specific class of brain-resident macrophages called microglial cells, which were protected from the clodronate liposomes treatment by the blood-brain barrier.

When tested in vitro, primary microglial cultures showed the ability to induce cytotoxicity in co-cultured tumour cells and such an effect was greatly enhanced by $\mathrm{CpG}-\mathrm{C}$. The authors observed an increase in both tumour cell apoptosis and phagocytosis capacity of microglial cells upon CpG-C treatment. Consistently, when tested in vivo, depletion of microglia or pharmacological inhibition of

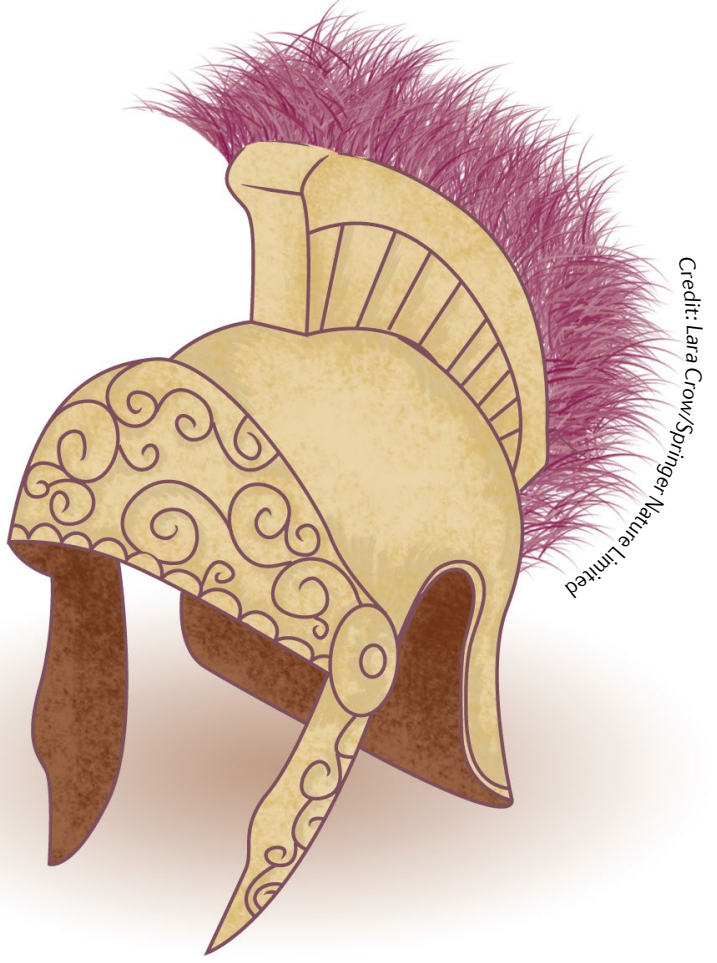

microglial activation abrogated the beneficial effects of CpG-C.

Using longitudinal intravital imaging and ImageStream fluorescence-activated cell sorting (FACS) analysis, the authors showed that microglial cells initiate phagocytic processes very early on during metastatic colonization. CpG treatment did not affect either the number of microglial cells or the number of braininfiltrating tumour cells. Instead, it enhanced the microglia-mediated phagocytosis of tumour cells at very early stages following tumour cell inoculation.

In conclusion, these data strongly suggest that use of the TLR9 agonist CpG-C might be a suitable approach for the prophylactic treatment of patients with cancer at high risk of developing brain metastasis and strongly encourage further testing in additional clinically relevant models to establish its translatability to the clinic.

Maria Giuseppina Baratta, Senior Editor, Nature Communications

of developing

brain

metastasis

ORIGINAL ARTICLE Benbenishty, A. et al.

Prophylactic TLR9 stimulation reduces brain metastasis through microglia activation. PLOS Biol.

https://doi.org/10.1371/journal.pbio.2006859 (2019) 